\title{
Clinical Performance of a New Hybrid Contact Lens for Keratoconus
}

\author{
Gonzalo Carracedo, Ph.D., José Manuel González-Méijome, Ph.D., Daniela Lopes-Ferreira, M.Sc., \\ Jesús Carballo, O.D., and Laura Batres, M.Sc.
}

\begin{abstract}
Objectives: To compare the clinical performance of the Clearkone hybrid contact lens for the treatment of keratoconus against the habitual contact lens of the patients.

Methods: A total of 33 eyes from 18 patients were fitted with the Clearkone. High- and low-contrast visual acuity (HCVA and LCVA), central corneal thickness (CCT), and contrast sensitivity acuity (CSF) were recorded with habitual lenses (prestudy visit) and after 1 week, 15 days, and 1 month of wear of prescribed Clearkone. Subjective vision and comfort were rated using visual analogue scales (VAS).

Results: Three patients discontinued the study, one because of diffuse corneal staining after 1 day of use and the other two because of extreme discomfort. The rest of the patients completed the 1-month study. High contrast visual acuity and LCVA (logMAR) improved significantly from $0.16 \pm 0.12$ and $0.44 \pm 0.22$, respectively, with the patient's habitual contact lenses to $-0.006 \pm 0.058$ and $0.23 \pm 0.13$ after 1 day wearing Clearkone, remaining significant during all follow-up visits $(P<0.001$; repeated measures analysis of variance [RM-ANOVA]). There were no statistically significant differences in the mean CCT. The improvement of CSF was statistically significant with hybrid contact lenses prescribed compared with the patient's habitual contact lenses $(P<0.001$; RM-ANOVA test). Improvement in VAS score, with prescribed Clearkone, was statistically significant for comfort ( $P=0.043$; RM-ANOVA test), but not for the subjective vision $(P=0.759$; RM-ANOVA test).

Conclusions: Clearkone provides an improvement in visual acuity, contrast sensitivity, and subjective comfort in patients with keratoconus when compared with other contact lens options. However, clinicians must get specific training to fit the lens and be aware of potential adverse events.
\end{abstract}

Key Words: Hybrid lens_-Comfort—Visual outcomes—Keratoconus.

(Eye \& Contact Lens 2013;0: 1-5)

From the Department of Optics II (Optometry and Vision) (G.C., J.C.), School of Optics, Universidad Complutense de Madrid, Madrid, Spain; Clinical \& Experimental Optometry Research Lab (J.M.G.-M., D.L.-F.), Center of Physics (Optometry), School of Sciences, University of Minho, Braga, Portugal; and Laboratorios Lenticon SA (G.C., L.B.), Madrid, Spain.

The authors have no financial interest on the materials and instruments used in this study. G. Carracedo is a part-time employee and L. Batres is a full-time employee of Laboratorios Lenticon SA and a distributor of Clearkone in Spain and Portugal. None of the authors have received funding to conduct this study.

Address correspondence and reprint requests to Gonzalo Carracedo, Faculty of Optic and Optometry, Department Optics II (Optometry and Vision), C/Arcos del Jalon 118 28032, Madrid, Spain; e-mail: gonzalocarracedo@gmail.com

Accepted XX XX, XXXX.

DOI: $10.1097 /$ ICL.0b013e3182a70ff2
K eratoconus is a progressive, asymmetric, dystrophy of the cornea characterized by steepening and distortion of the cornea, apical thinning, and central scarring. It is generally bilateral and progresses asymmetrically in both eyes of the same individual. ${ }^{1,2}$

Keratoconus is usually treated with contact lenses (CL), surgical procedures to overcome CL intolerance or to stop disease progression. ${ }^{3-5}$ Contact lenses that are most frequently used to compensate irregular astigmatism are currently made of gas-permeable (GP) materials in conventional spherical, aspheric designs, or other nonconventional designs. ${ }^{6-8}$ Despite rigid gas-permeable have shown to afford good visual performance, lens intolerance or physical damage to the corneal epithelium suggests that other approaches could be considered, using a diversity of materials, designs, and fitting approaches. $^{9,10}$

Hybrid lenses such as Saturn II and its evolution into SoftPerm in the 1990s had been an effective and convenient modality for providing satisfactory visual outcomes in keratoconic patients. ${ }^{11,12}$ However, adverse effects related with hypoxia have raised some concern in the clinical community with respect to these lenses. ${ }^{13-15}$

Currently, marketed hybrid lenses are made with high-Dk materials and incorporate designs that allow practitioners to determine proper fit of these lenses and achieve excellent outcomes in eyes regular astigmatism ${ }^{16}$ and also in irregular or distorted corneas. ${ }^{17}$ Moreover, specific designs targeted for use on ectatic corneas and eyes with keratoconus are now available. ${ }^{18}$ In 2008, an innovative hybrid contact lens for keratoconus was introduced which allows fitting of lenses with different sagittal heights to address the increasing corneal vaulting as ectasia evolves. The rigid portion of the lens is a reverse geometry design with a fixed chord length to vault over the cone and avoid apical corneal touch. This lens has proved to perform superiorly to its predecessors in oxygen transmission. ${ }^{19}$ However, some cases of severe complications, such as corneal edema or Acanthamoeba keratitis, have also been reported in the literature. ${ }^{20,21}$

When surgery is not an option, patients with keratoconus must wear CL to achieve acceptable visual performance for long periods of time. The aim of this study was to evaluate the visual function, the corneal thickness, and also, subjective comfort, handling, and subjective vision values after hybrid CL fitting in keratoconic patients in 1 day, 1 week, 2 weeks, and 1-month follow-up.

\section{METHODS}

\section{Subjects and Lenses}

Eighteen patients (13 male, 5 female; mean age $=29.45 \pm 5.13$ years old) from the Clinical and Experimental Optometry Research Laboratory (University of Minho, Braga, Portugal) and the Clinical 
Optometry of Madrid (University Complutense of Madrid, Madrid, Spain) were included in this study. The study protocol was approved by the ethics committee of the Universidad Complutense de Madrid and followed the tenets of the Declaration of Helsinki. Before enrollment, all patients were informed of the study duration, and required testing and all signed an informed consent. The demoT1 graphic characteristics of these patients are detailed in Table 1. All the patients were fitted with the Clearkone (SynergEyes, Inc., Carlsbad, CA) hybrid contact lens manufactured from rigid gaspermeable material (paflufocon D) and SynergEyes poly-HEMA material (hem-iberfilcon A) following the manufacturer's guidelines. Follow-up examinations were performed at 1 day, 1 week, 2 weeks, and 1 month after successful fitting of the lenses. The study was terminated at 1 month because this was intended to be a short-term study to evaluate the performance of the lenses, but was not intended to refit the patients who otherwise were successfully wearing other CL. High-contrast visual acuity in $\log$ MAR values (HCVA), low-contrast visual acuity in $\log$ MAR values (LCVA), contrast sensitivity in logarithmic units (CSF), and corneal pachymetry were measured with habitual CL (prestudy visit) and with prescribed hybrid CL. Finally, with prescribed hybrid CL, we were evaluated comfort and subjective vision using a subjective evaluation questionnaire (visual analogue scales [VAS]).

\section{Fitting Procedures}

The fitting of the Clearkone lens is based on the concept of sagittal depth in relation to the cornea. Proper fitting of the Clearkone depends on choosing a lens with sagittal depth that is sufficient to clear the elevation of the cone based on observation of the lens using sodium fluorescein. This is achieved by first determining the vault needed to clear the cone, and then, determining the skirt curvature, also through observations using sodium fluorescein, that fits onto the sclera to create an appropriate landing zone. The vault and the skirt curvature should be fit separately. The available parameters are included in Table 2.

Visual acuity (VA) and the contrast sensitivity (CS) were measured with habitual CL and prescribed Clearkone lenses under photopic $\left(85 \mathrm{~cd} / \mathrm{m}^{2}\right)$ luminance conditions. The acuity charts were externally illuminated with a halogen lamp behind a screen connected to a potentiometer to adjust the exact voltage needed to reach the adequate luminance level. This setup provides uniform luminance over the chart. Normal room lighting was left on. Measures of luminance for the tests were obtained using a MAVO-SPOT 2 USB luminance meter (Gossen Lighting Control).

TABLE 1. Demographic Information of the Population Enrolled in the Study

\begin{tabular}{lc}
\hline Parameter & Values \\
\hline Number of patients (eyes) & $18(33)$ \\
Mean age \pm SD, yr & $29.45 \pm 5.13$ \\
Age range, yr & 22,38 \\
Gender (male/female) & $13 / 5$ \\
Mean corneal astigmatism \pm SD, D & $-6.66 \pm 5.13$ \\
Range of corneal astigmatism, D & $0.70,16.80$ \\
Mean keratometry, D & \\
$\quad$ Flat & $48.46 \pm 7.33$ \\
Steep & $55.11 \pm 9.37$ \\
Mean refractive sphere \pm SD, D & $-5.45 \pm 3.83$ \\
Range of refractive sphere, D & $-0.75,-13.75$ \\
Mean refractive cylinder \pm SD, D & $2.78 \pm 1.89$ \\
Range of refractive cylinder, D & $0.50,-5.75$ \\
\hline
\end{tabular}

TABLE 2. Technical Details of the Contact Lenses Being Used and Parameters Fitted to Patients in this Study

\begin{tabular}{lc}
\hline Brand & Clearkone \\
\hline Manufacturer & SynergEyes \\
Material (RGP Center) & Paflufocon D \\
Material (soft skirt) & Hem-iberfilcon A \\
Water content (RGP center) & $<1 \%$ \\
Water content (soft skirt) & $27 \%$ \\
Oxygen permeability (RGP center) & $100^{a}$ \\
Oxygen permeability (soft skirt) & $9.3^{a}$ \\
$\mathrm{t}_{\mathrm{c}}$ mm & 0.18 to 0.39 \\
Power, D & 14.50 \\
Overall diameter, mm & 0.05 to 0.75 \\
Vault, mm & Steep medium and flat \\
Base curve radius (soft skirt) &
\end{tabular}

${ }^{a}$ Method for determination of oxygen permeability: ISO/DIS 9913.1 1994. Optics and Optical instruments - Contact Lenses, part 1: Determination of oxygen permeability and transmissibility with the Fatt method. (PHEMA Standard).

$\mathrm{RGP}$, rigid gas permeable.

To evaluate the VA, Bailey-Lovie letter charts were used, first using the high-contrast (96\%) side and then with the low-contrast $(10 \%)$ side at a viewing distance of $4 \mathrm{~m}$. Each letter read correctly on each line was given a score of $0.02 \mathrm{log}$ units. In this chart, a loss of one line of letters corresponds to a logMAR increase of $0.1 .^{22}$

Contrast sensitivity was determined using a Pelli-Robson letter chart (Clement Clarke International Ltd., Edinburgh, United Kingdom). This test is a quick, reliable, and repeatable method of evaluating CS. ${ }^{23,24}$ The chart consists of 16 triplets of $4.9 \times$ $4.9 \mathrm{~cm}$ letters. When viewed at $1 \mathrm{~m}$, the letters subtend $3^{\circ}$ equivalent to a 20/720 Snellen letter and a spatial frequency of approximately $1 \mathrm{c} /$ degree. ${ }^{5}$ Within each triplet, the letters have the same contrast, and the contrast in each successive triplet decreases by a factor of $0.15 \log$ units, ranging from 0.00 to $2.25 \mathrm{log}$ units. The tests were performed with a viewing distance at $1 \mathrm{~m}$, and two different letter charts were used to avoid the subjects being able to memorize the letters. We used the test's instruction for scoring the normal values, the logarithmic CS value of the last triplet. Results were recorded when at least 2 letters were correctly seen.

Corneal thickness was measured in the central using a portable AU6 (SP-100 Handy Pachometer Tomey, Nagoya, Japan) as described by Queirós. ${ }^{7}$ This portable pachymeter operates at $20 \mathrm{MHz}$ and measures thicknesses in the range of 150 to 1200 lumens at calibration speeds ranging from 1400 to $2000 \mathrm{~ms}$. One drop of $1 \%$ tetracaine hydrochloride was instilled before pachymetric readings were taken avoiding excessive compression of the tip probe against the cornea. The pachometer was calibrated before data acquisition at each measurement session. Calibration was accepted when five measurements of the test block were taken with an accuracy of $\pm 1 \mu \mathrm{m}$.

Visual analogue scales previously used to rate comfort with GP and soft $\mathrm{CL}^{25-27}$ were administered at each exam by asking patients to record their subjective impressions of vision and comfort using a scale from 0 (lowest) to 10 (highest). The scale was horizontally oriented, measuring exactly $10 \mathrm{~cm}$ and the value for statistical analysis was measured with a rule at the point where the mark inserted by the patient crossed the scale. During the study, patients also recorded their wearing time at 1, 2, 3, 5, 7, 14, 21, and 28 days after fitting. 


\section{Statistical Analysis}

Data were analyzed by statistical package SPSS version 15.0 for Windows (SPSS, Inc., Chicago, IL). The values presented are the means and SD for the each study variable. Normality of distribution was assessed using Shapiro-Wilk test. One-way repeated measure (RM-ANOVA) was used to assess the trend of the different parameters assessed in this study. $P<0.05$ was considered statistically significant.

\section{RESULTS}

All patients had been previously diagnosed with keratoconus, presenting grade II to grade III keratoconus according to the keratoconus severity score grading scale. ${ }^{28}$ Habitual correction for 3 patients ( 5 eyes) consisted of a thick soft CL design for keratoconus, 2 patients ( 3 eyes) were wearing GP corneo-scleral CL and the other 13 patients ( 25 eyes) were wearing aspheric GP CL for keratoconus. Three patients discontinued the study before 1 month after fitting of the Clearkone lens, one ( 1 eye) for presenting with diffuse corneal staining after 1 day of wear, 2 patients ( 4 eyes) discontinued after reporting significant discomfort after 4 to 5 hours of wear, and 2 patients discontinued because of difficulty with insertion of the lenses. Patients who completed the study showed a corneal staining grade 0 or 1 in all visits (the Cornea and Contact Lens Research Unit (CCLRU) grading scales was used).

Visual acuity outcomes presented herein are reported in $\log$ MAR values. Mean HCVA without correction was $0.72 \pm$ 0.29 , and mean LCVA without correction was $0.96 \pm 0.39$. When we performed overrefraction with their habitual CL and with prescribed Clearkone lenses, we did not find statistical improvement in HCVA and LCVA. As shown in Figure 1, both HCVA and LCVA with prescribed hybrid CL at the 1-day follow-up examination showed a statistically significant improvement compared with HCVA and LCVA with habitual CL (prestudy visit), which then remained fairly remained constant throughout the 1-month follow-up. The mean HCVA with hybrid CL at the end of the follow-up period was $-0.024 \pm 0.071(P<0.001)$. At the same period, the mean of the LCVA was $0.21 \pm 0.09(P<0.001)$

T3 (Table 3). All of the Clearkone lenses fit properly and centered well on the eyes.
Contrast sensitivity improved from $1.26 \pm 0.22$ logarithmic units with the habitual CL to $1.65 \pm 0.19$ after 1 -month of wear with the Clearkone lens. The improvement was statistically significant at all follow-up examinations ( $P<0.001$; RM-ANOVA test) (Table 3$)$.

Mean VAS scores for vision and comfort are given in Figure 2. Subjective scores for vision improved from $7.43 \pm 1.85$ at first day of wearing to $7.89 \pm 1.93$ after 1 month of the hybrid contact lens fitting. Maximum mean scores for vision were obtained at 15 days of follow-up, $8.24 \pm 1.93$, and mean comfort score rates improved from $7.41 \pm 0.81$ after the first day of wearing to $8.37 \pm 1.43$ after 1 month. Improvement in mean comfort scores was statistically significant ( $P=0.043$; RM-ANOVA test; Figure 2). But not for the subjective vision ( $P=0.759$; RM-ANOVA).

Pachymetry values remained constant throughout the study with AU8 no statistically significant changes compared to prefitting values. Mean pachymetry was $478.5 \pm 66.4 \mu \mathrm{m}$ on the first day and $480.1 \pm 68.5 \mu \mathrm{m}$ at the end of the follow-up period.

\section{DISCUSSION}

Gas-permeable lenses are often considered the gold standard for fitting keratoconus patients, with several studies showing their effectiveness and safety. ${ }^{29-32}$ The downside of GP CL is that many patients cannot tolerate gas-permeable lenses because of comfort issues. The SynergEyes Clearkone is the first hybrid CL with a specific design made for keratoconus patients comprising high-Dk materials.

The design of this hybrid lens allows better centration than that achieved with GP corneal lens, which as Guirao et al. ${ }^{33}$ reported as an important factor for the correction of the high-order aberrations values. This hybrid contact lens provides good VA and comfort during all-day wear, which is important to keratoconic patients who have no other means of satisfactory vision correction. This study is the first to report the visual outcomes and subjective response regarding this hybrid contact lens design.

The $83 \%$ of patients show an improvement in their high and LCVA with the prescribed hybrid CL compared with the patient's habitual lenses. This result is similar to the results reported by others. ${ }^{18}$ Although this result might be expected in patients wearing thick soft CL, the surprising result was that most of patients wearing GP lenses showed at least 1 line of increase in the HCVA achieved

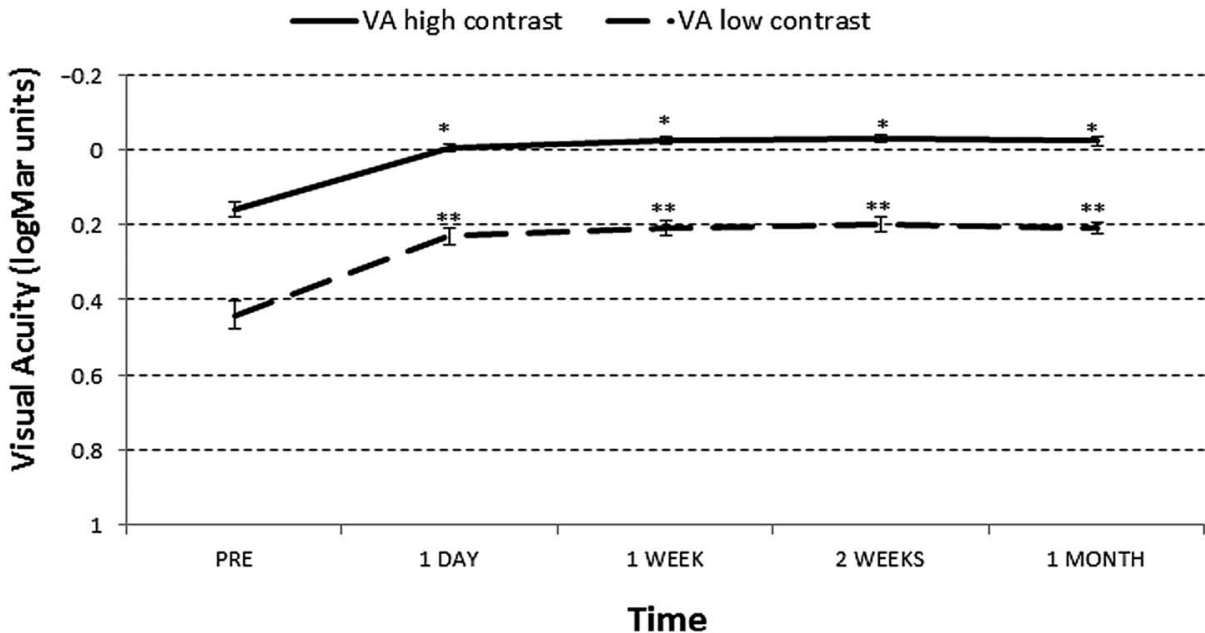

FIG. 1. Variation of high and low contrast visual acuity during all study. Errors bar presented in the figure are SEM to improve its comprehension. ${ }^{*} P$ values less than $0.05 \mathrm{VA}$ high contrast and ${ }^{* *} P$ values less than 0.05 for VA low contrast. 
TABLE 3. Values in the Prestudy Visit and Different Follow-up Visits During the Course of the Study

\begin{tabular}{lccc}
\hline Variable & Visit & Clearkone, mean (SD) & $P$ \\
\hline VA high contrast & Previsit & $0.16(0.12)$ & $<0.001$ \\
& $1 \mathrm{~d}$ & $-0.006(0.058)$ & \\
& $1 \mathrm{wk}$ & $-0.026(0.062)$ & \\
VA low contrast & $2 \mathrm{wk}$ & $-0.031(0.055)$ & \\
& $4 \mathrm{wk}$ & $-0.024(0.071)$ & \\
& Previsit & $0.44(0.22)$ & \\
& $1 \mathrm{~d}$ & $0.23(0.13)$ & \\
& $1 \mathrm{wk}$ & $0.21(0.11)$ & \\
& $2 \mathrm{wk}$ & $0.20(0.11)$ & \\
CSF & $4 \mathrm{wk}$ & $0.21(0.09)$ & \\
& Previsit & $1.26(0.22)$ & \\
& $1 \mathrm{~d}$ & $1.49(0.11)$ & \\
& $1 \mathrm{wk}$ & $1.52(0.17)$ & \\
& $2 \mathrm{wk}$ & $1.58(0.14)$ & \\
& $4 \mathrm{wk}$ & $1.65(0.19)$ & \\
& Previsit & $478.5(66.4)$ & \\
& $1 \mathrm{~d}$ & $475.6(62.1)$ & \\
& $1 \mathrm{wk}$ & $480.2(71.2)$ & \\
& $2 \mathrm{wk}$ & $482.3(69.4)$ & \\
& $4 \mathrm{wk}$ & $480.1(68.5)$ &
\end{tabular}

Previsit values are with habitual contact lenses by repeated measures analysis of variance

CSF, contrast sensitivity acuity; VA, Visual acuity.

with their previous lenses. This could be because of the excellent centration we observed with the hybrid CL compared with our experience with GP lenses. Furthermore, attempts to improve centration by changing base curve radius in GP induces VA changes. ${ }^{30}$

Pelli-Robson contrast acuity measurements are an appropriate and reliable tool for assessing vision related to changes in CS. Different studies have proposed Pelli-Robson contrast test as a reliable instrument to measure the visual performance. ${ }^{34,35}$ Although previous studies have demonstrated reduced CS in eyes with keratoconus, ${ }^{36}$ others have shown that hybrid CL and GP CL, improve CS as measured with Pelli-Robson test, to near-normal values. ${ }^{37}$ Our results showing improved CS with the Clearkone lens are consistent with these previous studies.

We used a VAS to evaluate comfort and subjective vision. This scale has previously been demonstrated to be a valid, responsive, and reliable evaluation. ${ }^{38}$ The subjective outcomes in this study show good ratings of subjective vision and comfort throughout the study which is consistent with the VA measured with the Balie-Lovie letter charts. In our study, subjective rating of comfort improved to $8.37 \pm 1.43$ at 1 month, which is similar to the results reported by other authors using the VAS but with soft toric CL. ${ }^{16}$ This provides strong evidence that many patients who are intolerant to GP lenses because of discomfort may find the Clearkone hybrid CL an acceptable alternative.

Despite the good clinical performance in visual rehabilitation, clinicians should be aware of potential complications with this fitting approach. These include corneal indentation in the transition zone between the rigid and the soft material, typically associated with a loss of vaulting overtime. Although a cause-effect relationship has not been established, this could be partially responsible for complications such as severe keratitis. ${ }^{21}$ In a recent study, the authors compared immunohistochemical changes induced by piggyback lens system and the Clearkone hybrid lens in patients with keratoconus during six months of wear. They evaluated proinflammatory molecules in tears, stromal keratocyte density, and goblet cell density and found no differences between these types of CL. ${ }^{39}$ These finding suggest that Clearkone is at least as safe as piggyback lenses for keratoconus patients.

Because of limited tear turnover, stagnation edema is also a potential complication, and epithelial edema has already been reported. ${ }^{20}$ However, other studies have shown that the oxygen availability behind modern hybrid lenses such as Clearkone might be much improved ${ }^{19}$ compared with other lenses for keratoconus such as the SoftPerm lens. ${ }^{14}$ Thus, despite of the case previously reported, edema-related complications seem to be unlikely with these lenses. Our results endorse this finding, showing stable central corneal thickness over the period of study.

Despite the initial comfort and apparent "easy of fitting," it is commonly accepted that learning curve is necessary with hybrid CL for keratoconus. ${ }^{16}$ Nonetheless, Shin et al. ${ }^{40}$ reported a success rate of $77.8 \%$ and Abdalla et al. ${ }^{18}$ reported a success rate of approximately $86 \%$ using hybrid lenses, which are similar to our $83 \%$ of patients who wore the hybrid lenses with good VA and comfort during the month of study. These authors also noticed that fitting of hybrid lenses was more likely to be successful in corneas with steeper K readings. Our results agree with this finding, and we believe this might be potentially related to the fact that eyes with steeper keratometry measurements tend to also have more severe ectasia,
FIG. 2. This figure showed the variation of subjective comfort and visual ratings during all study. Errors bar presented in the figure are SEM to improve its comprehension. ${ }^{*} P$ value less than 0.05 for comfort scores repeated measures analysis of variance.

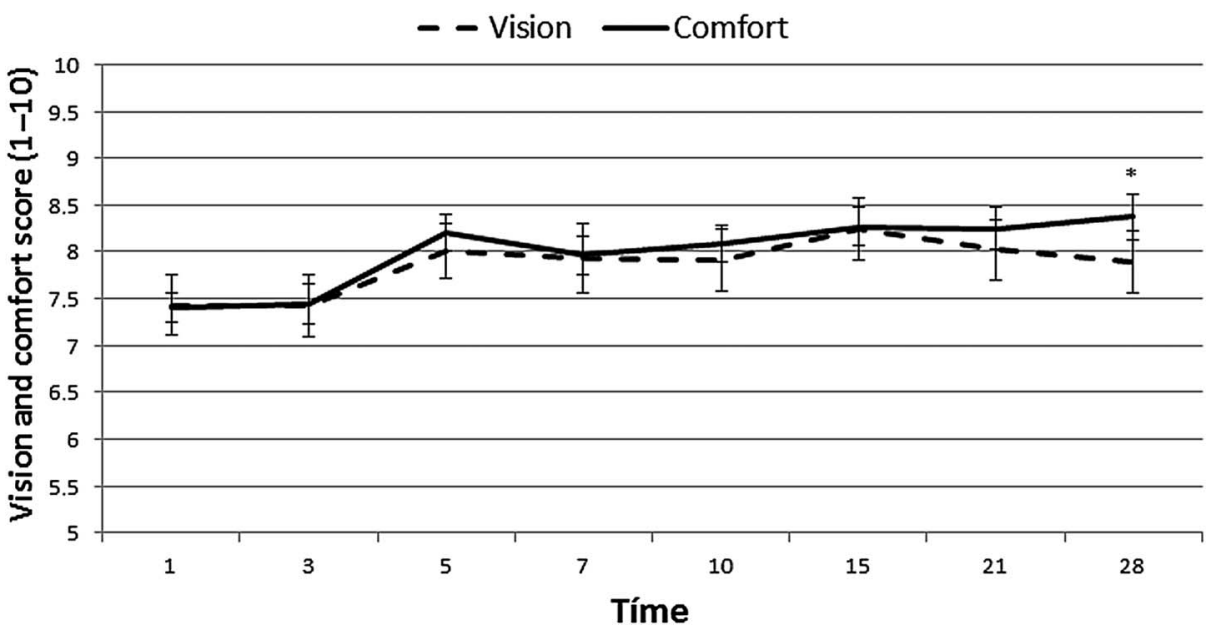

Eye \& Contact Lens • Volume 0, Number 0, Month 2013 
which may indicate a tendency toward intolerance to other forms of correction, making the patient more tolerant to hybrid lens fitting.

Two limitations of this study is the relatively brief follow-up examination period of one month and no control group evaluated. This study does not show how the lens behaves in the longer term, and particularly in aspects such as comfort, vision, and overall satisfaction over longer periods of time. These factors could be affected by the durability of lenses, care of lenses, how much cleaning and maintenance is necessary, the incidence of deposits, safety, or by adverse events such as microbial keratitis or Acanthamoeba infection. It would be necessary to conduct further long-term clinical studies with this hybrid contact lens to control the ocular integrity and to evaluate some possible complications. In our study, no keratitis or neovascularization were found in patients that completed the study. Moreover, these long-term clinical studies should be conduct with control group and a double-masked design.

In conclusion, the results from the present pilot study suggest that the Clearkone hybrid contact lens provides a VA improvement in patients with moderate-to-severe corneal ectasia compared with others contact lens options. However, clinicians must get specific training to fit the lens and be aware of potential complications similar to those for other CL for keratoconus.

\section{REFERENCES}

1. Rabinowitz YS. Keratoconus. Surv Ophthalmol 1998;42:297-319.

2. Romero-Jimenez M, Santodomingo-Rubido J, Wolffsohn JS. Keratoconus: A review. Cont Lens Anterior Eye 2010;33:157-166; quiz 205.

3. Mannis MJ, Zadnik K. Contact lens fitting in keratoconus. CLAOJ 1989;15: 282-289.

4. Lembach RG. Use of contact lenses for management of keratoconus. Ophthalmol Clin North Am 2003;16:383-394, vi.

5. Kubaloglu A, Sari ES, Cinar Y, et al. Intrastromal corneal ring segment implantation for the treatment of keratoconus. Cornea 2011;30:11-17.

6. Betts AM, Mitchell GL, Zadnik K. Visual performance and comfort with the Rose K lens for keratoconus. Optom Vis Sci 2002;79:493-501.

7. Edrington TB, Szczotka LB, Barr JT, et al. Rigid contact lens fitting relationships in keratoconus. Collaborative Longitudinal Evaluation of Keratoconus (CLEK) Study Group. Optom Vis Sci 1999;76:692-699.

8. Sorbara L, Mueller K. Effect of lens diameter on lens performance and initial comfort of two types of GP lenses for keratoconus: A pilot study. J Optom 2011;4:22-29.

9. Lopez-Alemany A, Gonzalez-Meijome JM, Almeida JB, et al. Oxygen transmissibility of piggyback systems with conventional soft and silicone hydrogel contact lenses. Cornea 2006;25:214-219.

10. Gonzalez-Meijome JM, Jorge J, de Almeida JB, et al. Soft contact lenses for keratoconus: Case report. Eye Contact Lens 2006;32:143-147.

11. Apsey DA, Barr JT. Corneal response and vision with the Softperm lens in simulated aircraft conditions. J Am Optom Assoc 1996;67:151-159.

12. Maguen E, Caroline P, Rosner IR, et al. The use of the SoftPerm lens for the correction of irregular astigmatism. CLAO J 1992;18:173-176.

13. Ozkurt Y, Oral Y, Karaman A, et al. A retrospective case series: Use of SoftPerm contact lenses in patients with keratoconus. Eye Contact Lens 2007;33:103-105.

14. Owens H, Watters G, Gamble G. Effect of softperm lens wear on corneal thickness and topography: A comparison between keratoconic and normal corneae. CLAO J 2002;28:83-87.

15. Chung CW, Santim R, Heng WJ, et al. Use of SoftPerm contact lenses when rigid gas permeable lenses fail. CLAO J 2001;27:202-208.
16. Lipson MJ, Musch DC. Synergeyes versus soft toric lenses: Vision-related quality of life. Optom Vis Sci 2007;84:593-597.

17. Nau AC. A comparison of synergeyes versus traditional rigid gas permeable lens designs for patients with irregular corneas. Eye Contact Lens 2008;34: 198-200

18. Abdalla YF, Elsahn AF, Hammersmith KM, et al. SynergEyes lenses for keratoconus. Cornea 2010;29:5-8.

19. Pilskalns B, Fink BA, Hill RM. Oxygen demands with hybrid contact lenses. Optom Vis Sci 2007;84:334-342.

20. Fernandez-Velazquez FJ. Severe epithelial edema in Clearkone SynergEyes contact lens wear for keratoconus. Eye Contact Lens 2011;37:381-385.

21. Lee WB, Gotay A. Bilateral Acanthamoeba keratitis in Synergeyes contact lens wear: Clinical and confocal microscopy findings. Eye Contact Lens 2010;36:164-169.

22. Williams MA, Moutray TN, Jackson AJ. Uniformity of visual acuity measures in published studies. Invest Ophthalmol Vis Sci 2008;49:4321-4327.

23. Elliott DB, Sanderson K, Conkey A. The reliability of the Pelli-Robson contrast sensitivity chart. Ophthalmic Physiol Opt 1990;10:21-24.

24. Reeves BC, Wood JM, Hill AR. Reliability of high- and low-contrast letter charts. Ophthalmic Physiol Opt 1993;13:17-26.

25. Fujita H, Sano K, Sasaki S, et al. Ocular discomfort at the initial wearing of rigid gas permeable contact lenses. Jpn J Ophthalmol 2004;48:376-379.

26. Gonzalez-Meijome JM, Carracedo G, Gonzalez-Perez J, et al. Comfort and vision scores at insertion and removal during 1 month of wear of Paragon CRT for corneal reshaping. Eye Contact Lens 2011;37:302-306.

27. Fonn D, Gauthier CA, Pritchard N. Patient preferences and comparative ocular responses to rigid and soft contact lenses. Optom Vis Sci 1995;72: $857-863$.

28. McMahon TT, Szczotka-Flynn L, Barr JT, et al. A new method for grading the severity of keratoconus: The Keratoconus Severity Score (KSS). Cornea 2006;25:794-800.

29. Barnett M, Mannis MJ. Contact lenses in the management of keratoconus. Cornea 2011;30:1510-1516.

30. Jinabhai A, Radhakrishnan H, O’Donnell C. Visual acuity and ocular aberrations with different rigid gas permeable lens fittings in keratoconus. Eye Contact Lens 2010;36:233-237.

31. Schirmbeck T, Paula JS, Martin LF, et al. [Efficacy and low cost in keratoconus treatment with rigid gas-permeable contact lens]. Arq Bras Oftalmol 2005;68:219-222.

32. Xie PY, Wang D, Yang LN, et al. The evaluation of visual quality in keratoconus eyes corrected by rigid gas-permeable contact lens [Article in Chinese]. Zhonghua Yan Ke Za Zhi 2005;41:1086-1091.

33. Guirao A, Cox IG, Williams DR. Method for optimizing the correction of the eye's higher-order aberrations in the presence of decentrations. $J \mathrm{Opt}$ Soc Am A Opt Image Sci Vis 2002;19:126-128.

34. Pesudovs K, Coster DJ. Penetrating keratoplasty for keratoconus: The nexus between corneal wavefront aberrations and visual performance. $J$ Refract Surg 2006;22:926-931.

35. Pesudovs K, Hazel CA, Doran RM, et al. The usefulness of Vistech and FACT contrast sensitivity charts for cataract and refractive surgery outcomes research. Br J Ophthalmol 2004;88:11-16.

36. Zadnik K, Mannis MJ, Johnson CA. An analysis of contrast sensitivity in identical twins with keratoconus. Cornea 1984;3:99-103.

37. Mantyjarvi M, Laitinen T. Normal values for the Pelli-Robson contrast sensitivity test. J Cataract Refract Surg 2001;27:261-266.

38. de Boer AG, van Lanschot JJ, Stalmeier PF, et al. Is a single-item visual analogue scale as valid, reliable and responsive as multi-item scales in measuring quality of life? Qual Life Res 2004;13:311-320.

39. Acar BT, Vural ET, Acar S. Effects of contact lenses on the ocular surface in patients with keratoconus: Piggyback versus ClearKone hybrid lenses. Eye Contact Lens 2012;38:43-48.

40. Shin A, Chang C, Fry K, et al. The use of hybrid contact lenses in keratoconus and ectasia patients post-intacs and collagen crosslinking. Invest Ophthalmol Vis Sci 2012;53:E-Abstract 4726. 